\title{
Growth Characteristics of V Factor-Independent Transformants of Haemophilus influenzae
}

\author{
HELEN M. WINDSOR, ROSA C. GROMKOVA, ${ }^{*}$ AND HENDRIK J. KOORNHOF \\ Emergent Pathogen Research Unit of the South African Medical Research Council, School of Pathology of \\ the University of the Witwatersrand and the South African Institute for Medical Research, 7 York Road, \\ Parktown, Johannesburg 2193, South Africa
}

\begin{abstract}
Haemophilus influenzae is a $\mathrm{V}$ factor-dependent species. A plasmid conferring $\mathrm{V}$ factor independence in Haemophilus parainftuenzae and Haemophilus ducreyi was transferred to plasmid-free $H$. influenzae Rd by DNA transformation. The growth characteristics of the transformants in a complex and a chemically defined medium were compared, and the ability of several exogenous pyridine nucleotides and precursors to support growth was examined. Although the transformants appeared to be $V$ factor independent in a complex medium, in a chemically defined medium they exhibited both $\mathbf{V}$ factor-dependent and nicotinamide-dependent growth. Because of the inability of the plasmid-free $H$. influenzae $R d$ to utilize nicotinamide for growth, it was concluded that the genes conferring this function were plasmid linked. Our results indicate that the $\mathrm{V}$ factor requirement, as it is presently defined, is not suitable to serve as a definitive taxonomic criterion for species determination in the family Pasteurellaceae.
\end{abstract}

Haemophilus influenzae, Haemophilus parainfluenzae, and several other members of the genus Haemophilus require $\mathrm{V}$ factor ( $\beta$-nicotinamide adenine dinucleotide [NAD]) for growth $(11,13)$. Unlike most bacteria, these species are not able to synthesize NAD de novo from low-molecularweight compounds but use a limited number of exogenous pyridine nucleotides or precursors as a source of NAD. Previous studies have shown that in addition to NAD, nicotinamide riboside (NR), $\beta$-nicotinamide mononucleotide (NMN), and NADP can serve as a V factor, while nicotinamide (NAm), nicotinic acid (NA), and quinolinic acid (QA) cannot $(3,4)$. The $\mathrm{V}$ factor requirement and the requirement for $\mathrm{X}$ factor have been used for many years as a basis for the classification and the laboratory identification of species from the genus Haemophilus.

Four unusual naturally occurring $\mathrm{V}$ factor-independent $H$. parainfluenzae isolates were identified in our laboratory a few years ago $(5,6)$. The genes coding for $V$ factor independence were located on a small 5.25-kb plasmid (24). Plasmids of a similar size conferring the ability to grow in the absence of $\mathrm{V}$ factor were recovered recently from Haemophilus ducreyi (25). This species is presently classified as a V factor-independent Haemophilus species, but DNA homology studies and physiological differences between $H$. ducreyi and other Haemophilus species have recently been used to question its inclusion as a Haemophilus species.

In this report we present data on the growth characteristics of $\boldsymbol{H}$. influenzae Rd transformants in the presence of a plasmid conferring $\mathrm{V}$ factor independence. Attempts were made to determine whether the plasmid confers the ability to utilize an exogenous pyridine nucleotide precursor (such as NAm) for growth. It is known that NAm can be used as a source of NAD by other $V$ factor-independent members of the genus Haemophilus (10). Because of the extrachromosomal location of the genes coding for $\mathrm{V}$ factor independence, we conclude that this characteristic is not suitable to serve as a major species-determining taxonomic criterion.

* Corresponding author.

\section{MATERIALS AND METHODS}

Bacterial strains and media. The strains used in this study are listed in Table 1 . The $H$. parainfluenzae isolates were grown in brain heart infusion (BHI) broth and BHI agar (Becton Dickinson) unsupplemented with NAD. For the growth of $H$. influenzae Rd the above-mentioned media were supplemented with $10 \mu \mathrm{g}$ of hemin (Sigma) per $\mathrm{ml}$ and $2 \mu \mathrm{g}$ of NAD (Sigma) per ml. The V factor-independent transformants of $H$. influenzae $\mathrm{Rd}$ were grown in hemin-supplemented BHI broth and agar as well as in the chemically defined medium (CDM) developed by Herriott et al. (8). The following pyridine compounds supplied by Sigma were added to CDM to examine their ability to support the growth of the V-factor-independent transformants of $H$. influenzae Rd: NAD, NMN, NAm, NA, nicotinic acid mononucleotide (NAMN), nicotinic acid adenine dinucleotide (NAAD), and QA. The $H$. ducreyi strains were grown on the enriched agar medium described by Nsanze et al. (16) in $5 \% \mathrm{CO}_{2}$ at $35^{\circ} \mathrm{C}$ except the type strain, CIP $542^{\mathrm{T}}$, which was grown on chocolate agar.

Growth in CDM. Frozen BHI cultures of $H$. influenzae $\mathrm{Rd}$ or the $\mathrm{V}$ factor-independent transformants were diluted 1:50 in 10-ml aliquots of hemin-supplemented BHI broth in 250-ml flasks. These cultures were incubated for about $5 \mathrm{~h}$ at $37^{\circ} \mathrm{C}$ with agitation at $200 \mathrm{rpm}$ on a gyratory shaker until they reached an optical density at $650 \mathrm{~nm}$ of 1.0 . The cells were harvested by centrifugation, washed twice with $10 \mathrm{ml}$ of $\mathrm{CDM}$, and resuspended in $10 \mathrm{ml}$ of CDM. Aliquots of CDM in $500-\mathrm{ml}$ flasks were inoculated with the resuspended cells $(0.2 \mathrm{ml}$ in $20 \mathrm{ml}$ of CDM $)$ and incubated for $5 \mathrm{~h}$ in the absence of pyridine compounds. The filter-sterilized pyridine compounds were added to the starved cells to give a final concentration of $5 \mu \mathrm{M}$, and incubation was continued as described above. Samples were taken at different intervals and plated onto hemin-supplemented BHI agar to determine the number of CFU per ml over a 20 -h period.

Growth in complex medium. The inoculum was prepared as described in the previous paragraph. Duplicate $20-\mathrm{ml}$ aliquots of hemin-supplemented BHI broth, one with $5 \mu \mathrm{M}$ NAD added and one without any NAD, were inoculated with $0.2 \mathrm{ml}$ of washed and resuspended cells and incubated 
TABLE 1. Strains used in this study ${ }^{a}$

\begin{tabular}{llll}
\hline Species and strain & Plasmid & Site of isolation & $\begin{array}{c}\text { Source or } \\
\text { reference }\end{array}$ \\
\hline $\begin{array}{llll}\text { H. parainfluenzae } \\
9\end{array}$ & & & \\
15 & pHP9 & Sputum & SAIMR (6) \\
19 & pHP15 & Purulent skin lesion & SAIMR (6) \\
78 & pHP19 & Throat swab & SAIMR (6) \\
H. ducreyi & pHP78 & Genital ulcer & SAIMR (6) \\
ATCC 27722 & pHD27722 & Genital ulcer & ATCC \\
CIP 542 & pHD542 & Genital ulcer & ATCC \\
124 & pHD124 & Genital ulcer & SAIMR (25) \\
Y78 & pHDY78 & Genital ulcer & SAIMR (25) \\
C147 & pHDC147 & Genital ulcer & L. Slaney \\
H. influenzae Rd & & & G. Leidy \\
\hline
\end{tabular}

${ }^{a}$ All strains except $H$. influenzae $\mathrm{Rd}$ are $\mathrm{V}$ factor independent. ATCC, American Type Culture Collection, Rockville, Md.; CIP, Collection de l'Institut Pasteur, Paris, France; SAIMR, South African Institute for Medical Research, Johannesburg.

${ }^{b}$ L. Slaney, University of Manitoba, Winnipeg, Manitoba, Canada.

${ }^{c}$ G. Leidy, Columbia University, New York, N.Y.

at $37^{\circ} \mathrm{C}$ with shaking at $200 \mathrm{rpm}$. The number of CFU per $\mathrm{ml}$ was determined as described above.

DNA transformation. Purified plasmid DNA was prepared by the alkaline lysis method described by Sambrook et al. (18) with the addition of a phenol-chloroform extraction step. Competence in $H$. influenzae Rd was induced by the static aerobic procedure of Gromkova et al. (7). For the transformation assay, $0.1 \mathrm{ml}$ of competent cells and $0.8 \mathrm{ml}$ of BHI broth supplemented with NAD, hemin, and $1 \mathrm{mM} \mathrm{Mg}$ $\mathrm{SO}_{4}$ were added to $0.1 \mathrm{ml}$ of purified DNA at a saturating concentration of $1 \mu \mathrm{g} / \mathrm{ml}$. This mixture was incubated for $5 \mathrm{~h}$ at $37^{\circ} \mathrm{C}$, and then the culture was diluted and plated in triplicate on BHI agar unsupplemented with NAD for selection of the transformants. Control cultures with no added DNA were tested to check for spontaneous mutants.

\section{RESULTS}

Transformation of $H$. influenzae $R d$ to $\mathrm{V}$ factor independence. Plasmids conferring $\mathrm{V}$ factor independence in $H$. parainfluenzae and $H$. ducreyi were transferred to a plasmidfree $H$. influenzae Rd strain by DNA transformation. $H$. influenzae $\mathrm{Rd}$ is a nonencapsulated (rough) segregant of an encapsulated (smooth) serotype $d$ strain. It was chosen as a recipient because it is highly transformable (1) and it is able to grow in CDM. This medium was developed by Herriott et al. (8) specifically for $H$. influenzae Rd. Growth in this medium is important because it allows the determination of exogenous pyridine compounds required for growth. Four naturally occurring $\mathrm{V}$ factor-independent isolates of $H$. parainfluenzae and five $H$. ducreyi strains (two reference and three clinical) were used as a source of plasmid DNA (Table 1). Transformants were selected for their ability to grow on BHI agar without the addition of NAD. Each transformant acquired a small $5.25-\mathrm{kb}$ plasmid. Fig. 1 presents the electrophoretic mobility of two representative plasmids acquired by transforming $H$. influenzae Rd with plasmid DNA from $H$. parainfluenzae 9 and $H$. ducreyi ATCC 27722. Details on the transforming activity and other properties of the plasmid conferring $\mathrm{V}$ factor independence are described elsewhere $(24,25)$.

Kinetics of growth of the $V$ factor-independent transformants of $\boldsymbol{H}$. influenzae $R d$ in a complex medium. To study whether the presence of a plasmid conferring $\mathrm{V}$ factor

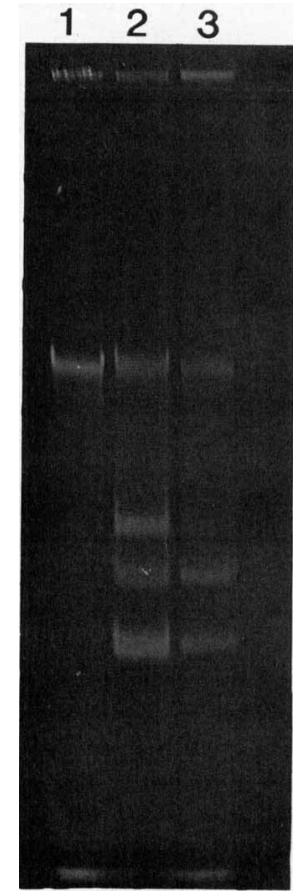

FIG. 1. Agarose gel electrophoresis of plasmid DNA preparations from $\mathrm{V}$ factor-independent $H$. influenzae Rd transformants. Lane 1, plasmid-free $H$. influenzae Rd used as the transformation recipient; lane 2, transformant of $H$. influenzae $\mathrm{Rd}$ using $\mathrm{V}$ factorindependent $H$. parainfluenzae 9 as the plasmid DNA donor; lane 3 , transformant of $H$. influenzae Rd using $H$. ducreyi ATCC 27722 as the plasmid DNA donor.

independence had an effect on the kinetics of growth of $H$. influenzae $\mathrm{Rd}$ in a complex medium, one $\mathrm{V}$ factor-independent transformant of $H$. influenzae Rd obtained by using DNA from each of the donor strains listed in Table 1 was examined for its ability to grow in BHI broth in both the presence and absence of NAD. Figures $2 a$ and $b$ present the growth curves of two representative transformants of $H$. influenzae Rd. Although the source of the plasmid in these transformants was different (Table 1), their growth curves exhibited similar profiles. Because of their ability to grow equally well in BHI broth in the presence and absence of $\mathrm{NAD}$, the transformants were designated $\mathrm{V}$ factor independent. The growth curves of the remaining seven transformants were similar to those presented in Fig. $2 a$ and $b$. The generation times of all transformants in unsupplemented and NAD-supplemented broth were in the range of 38 to $45 \mathrm{~min}$ and 40 to $51 \mathrm{~min}$, respectively. The generation time was determined by using the linear portion of the growth curve. Unlike the transformants, the isogenic plasmid-free $H$. influenzae Rd was not able to grow in the absence of NAD (Fig. 2c). The generation time of this strain in NAD-supplemented broth was $45 \mathrm{~min}$.

It has been previously demonstrated that spontaneous loss of the plasmid conferring $\mathrm{V}$ factor independence in $H$. influenzae Rd transformants occurs at a frequency of 0.1 to $0.2 \%$ of the progeny of a single clone (24). Our results show that the segregation to $\mathrm{V}$ factor dependence does not appear to have an effect on the growth curves in the absence of NAD (Fig. 2a and b).

Kinetics of growth of the $\mathrm{V}$ factor-independent transformants of $\boldsymbol{H}$. influenzae Rd in CDM. Two approaches have 

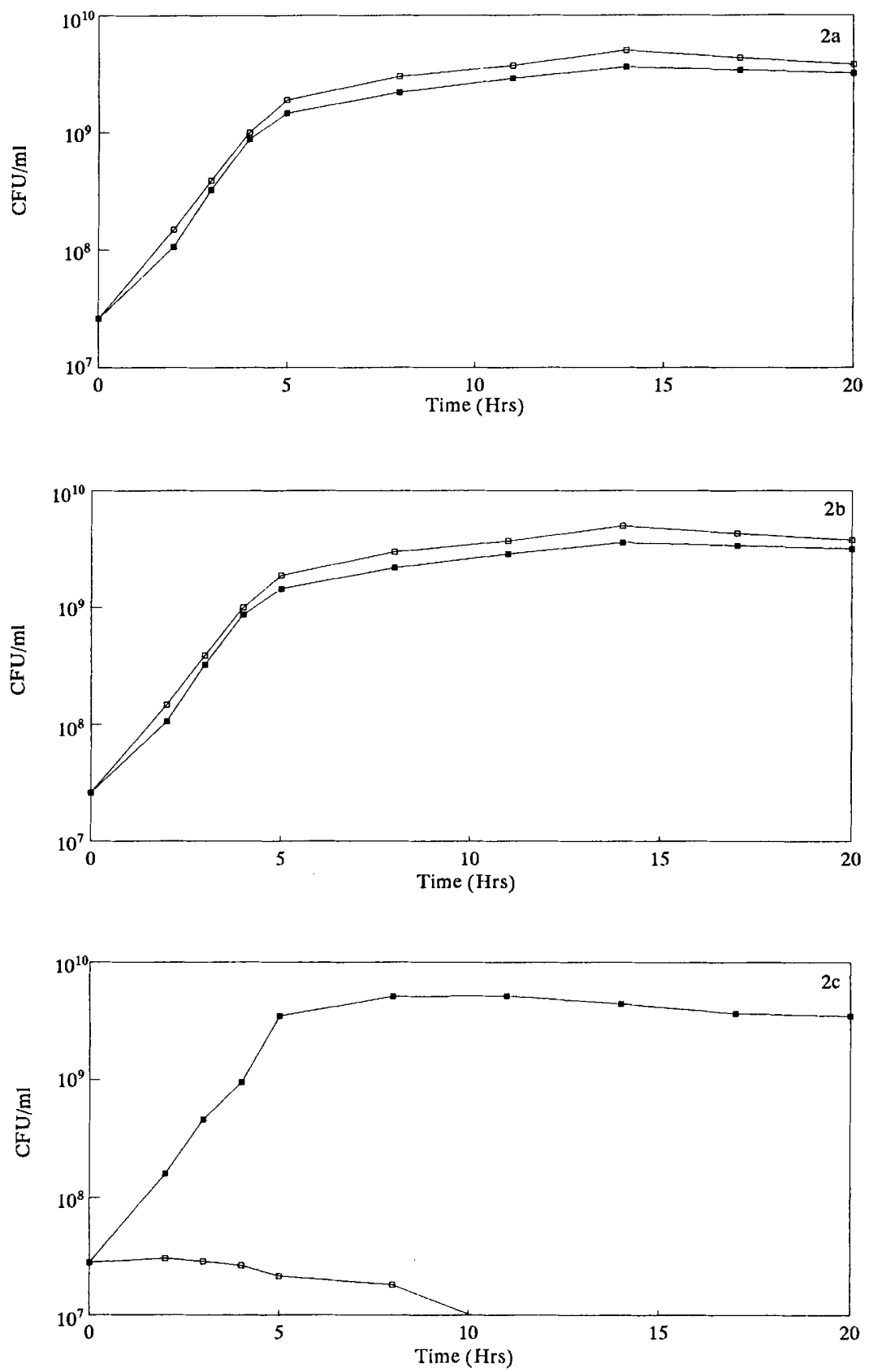

FIG. 2. Growth curves of $H$. influenzae Rd and V factor-independent transformants in BHI broth in the absence ( $\square)$ and presence $(\square)$ of $5 \mu \mathrm{M}$ NAD. (a) Transformant with pHP9; (b) transformant with pHD27722; (c) plasmid-free $H$. influenzae Rd.

previously been used for studying the biosynthetic pathway of NAD and the requirement for exogenous pyridine nucleotides. The first approach is to examine cell extracts for their ability to synthesize NAD and to identify the enzyme activities required for this synthesis $(3,9)$. The second approach, used in the present study, is to simply test exogenous pyridine nucleotides or precursors for their ability to support growth in a CDM (14). The pyridine compounds employed in this study were added to the CDM after $5 \mathrm{~h}$ of starvation of the cells. No increase in the optical density of the cells was observed during the starvation period. All nine $H$. influenzae Rd transformants tested were able to grow in the presence of NAD, NMN, and NAm, and their growth curves showed similar profiles. The growth curves of two representative $\mathrm{V}$ factor-independent transformants and those of the plasmid-free $H$. influenzae $\mathrm{Rd}$ in the presence and absence of exogenous pyridine compounds are presented in Fig. 3a, b, and c, respectively. Generation times of transformants in the presence of pyridine compounds were 51 to 63 min with NAD, 53 to 65 min with NMN, and 55 to 63 min with NAm. Unlike the transformants, the plasmid-free $H$. influenzae $\mathrm{Rd}$ failed to grow in the presence of NAm; however, NAD and NMN did support its growth (Fig. 3c). The generation time of $H$. influenzae $\mathrm{Rd}$ was 60 min in the presence of either NAD or NMN. Neither the plasmid-free $H$. influenzae Rd nor the transformants carry- 

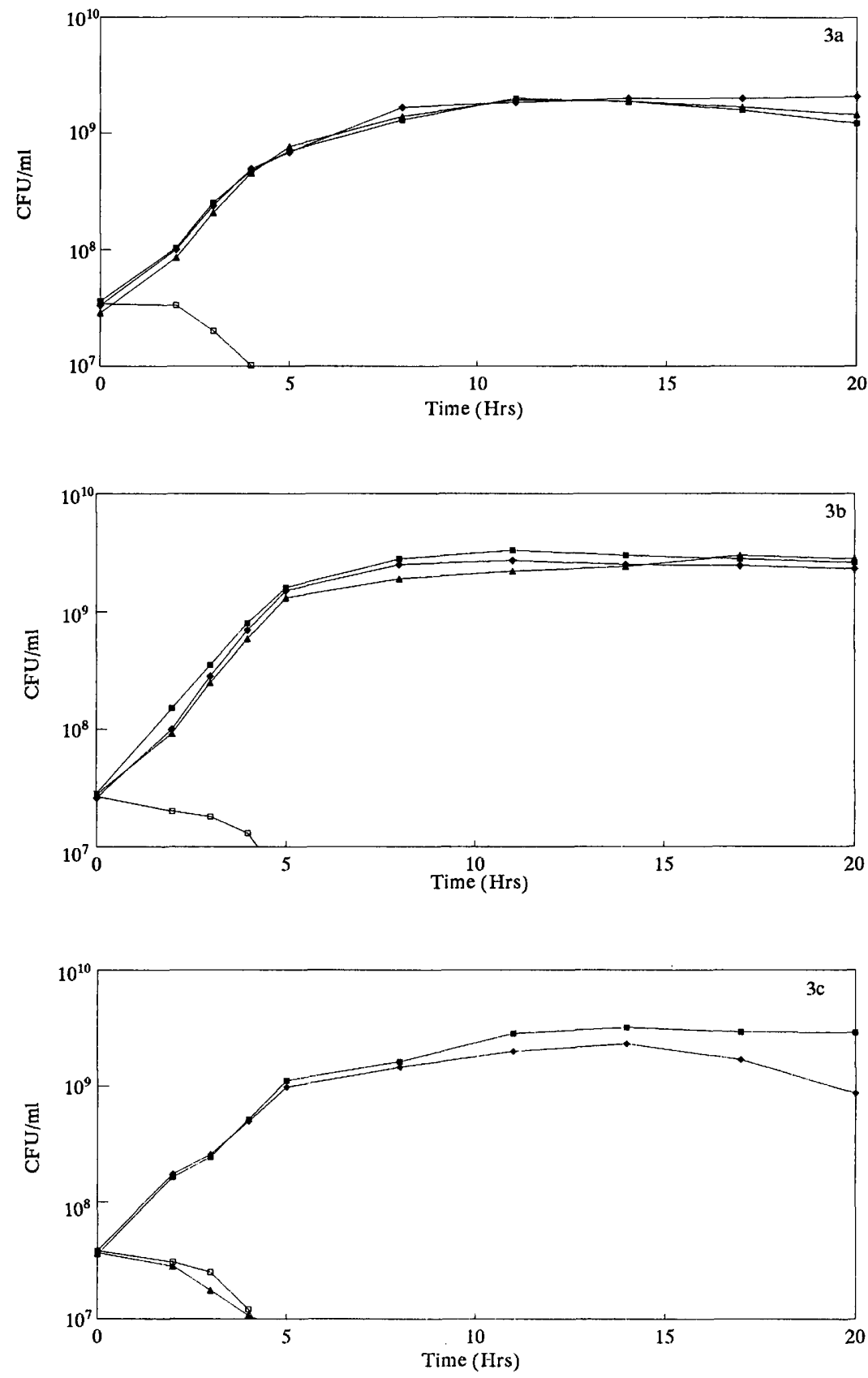

FIG. 3. Growth curves of $H$. influenzae Rd and V factor-independent transformants in CDM supplemented with NAD ( $\square)$, NMN $(\checkmark)$, or NAm (A) or unsupplemented ( $\square$ ). (a) Transformant with pHP9; (b) transformant with pHD27722; (c) plasmid-free $H$. influenzae Rd.

ing the $5.25-\mathrm{kb}$ plasmid were able to grow in unsupplemented CDM or in CDM containing NAAD, NAMN, NA, or QA (data not presented in Fig. 3a, b, or c), suggesting that these strains were incapable of de novo NAD biosynthesis.

It is of interest that in CDM the $H$. influenzae Rd transformants exhibit V factor-dependent growth (Fig. 3a and b), whereas in BHI broth their growth appears to be $\mathrm{V}$ factor independent (Fig. 2a and b). This was probably due to the presence of NAm in BHI broth (14). The growth in CDM of two $H$. influenzae Rd transformants that have lost the small plasmid was also examined. The growth curves of these isolates (data not presented) were similar to those of the original plasmid-free $H$. influenzae $\mathrm{Rd}$ recipient strain (Fig. 3c). The correlation between the loss of the plasmid and the loss of the ability to utilize NAm for growth supports the conclusion that the genes coding for utilization of NAm are plasmid linked.

\section{DISCUSSION}

Previous study on the biosynthetic pathway of NAD of the $\mathrm{V}$ factor-independent species Haemophilus haemoglobinophilus has revealed that NAm is the sole source of NAD in this organism and that NAD is synthesized directly from NAm via NMN (10). Recent investigation on the growth requirements in CDM of Actinobacillus pleuropneumoniae 
biotype 2 has provided evidence that this biotype shows both $\mathrm{V}$ factor-dependent and NAm-dependent growth (14). However, in a complex medium $A$. pleuropneumoniae biotype 2 appears to be $\mathrm{V}$ factor independent (17). Data presented in this paper show that the growth requirements in CDM of $H$. influenzae $\mathrm{Rd}$ transformants that have acquired the small $5.25-\mathrm{kb}$ plasmid from $H$. parainfluenzae and $H$. ducreyi donors are similar to those of $A$. pleuropneumoniae biotype 2 (Fig. $3 \mathrm{a}$ and $\mathrm{b}$ ) while the plasmid-free $H$. influenzae $\mathrm{Rd}$ (Fig. 3c), like $A$. pleuropneumoniae biotype 1 , is able to utilize only $\mathrm{V}$ factor for growth (14). In both systems the only difference in the growth of the $\mathrm{V}$ factor-dependent and the $\mathrm{V}$ factor-independent strains is the ability of the latter to utilize NAm. This suggests that the genetic differences between the $\mathrm{V}$ factor-dependent and the $\mathrm{V}$ factor-independent strains are not as significant as has been previously thought. Other examples of $\mathrm{V}$ factor-independent bacteria of the family Pasteurellaceae that require NAm for growth are Pasteurella multocida (12), Pasteurella haemolytica $(19,20)$, and Pasteurella ureae $(21,22)$. $P$. multocida has also been shown to exhibit $\mathrm{V}$ factor-dependent growth (2). These data illustrate that, within the family Pasteurellaceae, the requirement for exogenous pyridine nucleotides or precursors is not confined to the genus Haemophilus but, rather, may be characteristic of the family as a whole (15).

No previous information about the location of the genes involved in NAD biosynthesis in members of the family Pasteurellaceae is available. Four unusual V factor-independent $H$. parainfluenzae isolates (Table 1 ) were the first bacterial strains in which the genes coding for $\mathrm{V}$ factor independence were located on a small $5.25-\mathrm{kb}$ plasmid (Fig. 1) (24). Subsequently, a plasmid of a similar size also conferring $\mathrm{V}$ factor independence was recovered from $H$. influenzae Rd transformants with $H$. ducreyi DNA (Fig. 1) (25). In this paper we present evidence that the $\mathrm{V}$ factor independence is due to the ability of the cells to utilize NAm for growth and that the genes coding for this function are plasmid linked in $H$. parainfluenzae and $H$. ducreyi. Because of the small size of this plasmid, NAD is most probably synthesized directly from NAm by a pathway similar to that described for $H$. haemoglobinophilus (10). It would be of interest to determine whether the genes coding for $\mathrm{V}$ factor independence in other members of the family Pasteurellaceae are also plasmid linked. Although no published information about the location of the genes coding for the utilization of $\mathrm{V}$ factor is available, they appear to be of chromosomal nature because of their stability.

The recovery from natural sources of both $\mathrm{V}$ factordependent and $\mathrm{V}$ factor-independent isolates from species belonging to two different genera, Haemophilus (6) and Actinobacillus (17), indicates that the $\mathrm{V}$ factor requirement is variable among strains of the same species and is not suitable as a taxonomic criterion for species determination. In addition, the demonstration that in at least two Haemophilus species the genes conferring $\mathrm{V}$ factor independence are plasmid linked $(24,25)$ suggests that other $V$ factordependent species have the potential to become $\mathrm{V}$ factor independent because of the mobility of extrachromosomal genes or conversely, some $\mathrm{V}$ factor-independent isolates may become $\mathrm{V}$ factor dependent because of loss of the plasmid. We were able to demonstrate transfer of the plasmid conferring $\mathrm{V}$ factor independence between both homologous and heterologous species by DNA transformation (6, 24).

In a recent review Niven and O'Reilly analyzed the biochemical basis of the $\mathrm{V}$ factor requirement in three genera of the family Pasteurellaceae, Haemophilus, Pasteurella, and Actinobacillus, and concluded that a common characteristic of these genera is the lack of de novo NAD biosynthesis and the ability to grow in the presence of a few exogenous pyridine nucleotides or precursors (15). The authors propose the inclusion of NAm in the pyridine compounds that may serve as a $\mathrm{V}$ factor. This will allow the classification of all members of the family Pasteurellaceae as $V$ factor dependent. We agree with the opinion of Niven and $O$ 'Reilly that the definition of the $\mathrm{V}$ factor needs to be revised. However, in view of the extrachromosomal nature of the genes coding for NAm utilization in at least two Haemophilus species, we feel that it would be more appropriate to preserve the old definition of $\mathrm{V}$ factor and to include NAm as an additional pyridine compound that can be utilized for growth by any member of the family Pasteurellaceae. Thus a member of this family can be $\mathrm{V}$ factor dependent or NAm dependent or both. Because of the presence of NAm and other pyridine compounds in complex media, these media are not suitable for the determination of the growth requirements. They can be used only for initial screening of species of Haemophilus and Actinobacillus that are presently classified as $\mathrm{V}$ factor dependent, and if a strain appears to be $\mathrm{V}$ factor independent, it should be tested in synthetic or semisynthetic media for proper determination of the growth requirements. Although the frequency of the naturally occurring $\mathrm{V}$ factor-independent $H$. parainfluenzae isolates appears to be low (23), one should be aware of the possible spread of the plasmid conferring $V$ factor independence to other $\mathrm{V}$ factor-dependent species. According to present laboratory practice, isolates that are $\mathrm{V}$ factor independent cannot be identified as $H$. parainfluenzae or $H$. influenzae. This definition could interfere with the identification of strains that may be clinically important.

To satisfy the taxonomist, the microbial physiologist, and the clinical microbiologist, this question should be further debated in order to find a proper definition for $\mathrm{V}$ factor.

\section{ACKNOWLEDGMENTS}

We thank G. Leidy, L. Slaney, R. Ballard, Y. Dangor, and J. Saunders for kindly providing us with strains.

This research was supported by a grant from the South African Institute for Medical Research.

\section{REFERENCES}

1. Alexander, H. E., and G. Leidy. 1951. Determination of inherited traits of Haemophilus influenzae by deoxyribonucleic acid fractions isolated from type specific cells. J. Exp. Med. 93:345349.

2. Berkman, S. 1942. Accessory growth factor requirements of the members of the genus Pasteurella. J. Infect. Dis. 71:201-211.

3. Cynamon, M. H., T. B. Sorg, and A. Patapow. 1988. Utilization and metabolism of NAD by Haemophilus parainfluenzae. J. Gen. Microbiol. 134:2789-2799.

4. Foster, J. W., and A. G. Moat. 1980. Nicotinamide adenine dinucleotide biosynthesis and pyridine nucleotide cycle metabolism in microbial systems. Microbiol. Rev. 44:83-105.

5. Gromkova, R., Y. Dangor, and S. D. Miller. 1989. V-factor (NAD)-independent Haemophilus parainfluenzae recovered from a human genital ulcer. Eur. J. Clin. Microbiol. Infect. Dis. 8:569. (Letter.)

6. Gromkova, R., and H. J. Koornhof. 1990. Naturally occurring NAD-independent Haemophilus parainfluenzae. J. Gen. Microbiol. 136:1031-1035.

7. Gromkova, R. C., P. B. Rowji, and H. J. Koornhof. 1989. Induction of competence in nonencapsulated and encapsulated strains of Haemophilus influenzae. Curr. Microbiol. 19:241-245. 8. Herriott, R. M., E. Y. Meyer, M. Vogt, and M. Modan. 1970. 
Defined medium for growth of Haemophilus influenzae. J. Bacteriol. 101:513-516.

9. Kahn, D. W., and B. M. Anderson. 1986. Characterization of Haemophilus influenzae nucleotide pyrophosphatase. An enzyme of critical importance for growth of the organism. J. Biol. Chem. 261:6016-6025.

10. Kasarov, L. B., and A. G. Moat. 1973. Biosynthesis of NAD in Haemophilus haemoglobinophilus. Biochim. Biophys. Acta 320:372-378.

11. Kilian, M. 1976. A taxonomic study of the genus Haemophilus with the proposal of a new species. J. Gen. Microbiol. 93:9-62.

12. Koser, S. A., S. Berkman, and A. Dorfman. 1941. Comparative activity of nicotinic acid and nicotinamide as growth factors for microorganisms. Proc. Soc. Exp. Biol. Med. 47:504-507.

13. Lwoff, A., and M. Lwoff. 1937. Studies on codehydrogenases. I. Nature of growth factor "V." Proc. R. Soc. London B Biol. Sci. 122:352-359.

14. Niven, D. F., and M. Lévesque. 1988. V-factor-dependent growth of Actinobacillus pleuropneumoniae biotype 2 (Bertschinger 2008/76). Int. J. Syst. Bacteriol. 38:319-320.

1.5. Niven, D. F., and T. O'Reilly. 1990. Significance of V-factor dependency in the taxonomy of Haemophilus species and related organisms. Int. J. Syst. Bacteriol. 40:1-4.

16. Nsanze, H., F. A. Plummer, A. B. N. Maggwa, G. Maitha, J. Dylewski, P. Piot, and A. R. Ronald. 1984. Comparison of media for the primary isolation of Haemophilus ducreyi. Sex. Transm. Dis. 11:6-9.

17. Pohl, S., H. U. Bertschinger, W. Frederiksen, and W. Mann- heim. 1983. Transfer of Haemophilus pleuropneumoniae and the Pasteurella haemolytica-like organism causing porcine necrotic pleuropneumonia to the genus Actinobacillus (Actinobacillus pleuropneumoniae comb. nov.) on the basis of phenotypic and deoxyribonucleic acid relatedness. Int. J. Syst. Bacteriol. 33:510-514.

18. Sambrook, J., E. F. Fritsch, and T. Maniatis. 1989. Molecular cloning: a laboratory manual, 2nd ed. Cold Spring Harbor Laboratory, Cold Spring Harbor, N.Y.

19. Wessman, G. E. 1965. Cultivation of Pasteurella haemolytica in a casein hydrolysate medium. Appl. Microbiol. 13:426-431.

20. Wessman, G. E. 1966. Cultivation of Pasteurella haemolytica in a chemically defined medium. Appl. Microbiol. 14:597-602.

21. Wessman, G. E., and G. Wessman. 1970. Chemically defined media for Pasteurella multocida and Pasteurella ureae, and a comparison of their thiamine requirements with those of Pasteurella haemolytica. Can. J. Microbiol. 16:751-757.

22. Wessman, G. E., and G. Wessman. 1972. Requirements for growth of Pasteurella ureae in a chemically defined medium. Can. J. Microbiol. 18:107-109.

23. Windsor, H. M. Unpublished data.

24. Windsor, H. M., R. C. Gromkova, and H. J. Koornhof. 1991. Plasmid-mediated NAD independence in Haemophilus parainfluenzae. J. Gen. Microbiol. 137:2415-2421.

25. Windsor, H. M., R. C. Gromkova, and H. J. Koornhof. 1993. Transformation of $\mathrm{V}$-factor independence from Haemophilus ducreyi to Haemophilus influenzae and Haemophilus parainfluenzae. Med. Microbiol. Lett. 2:159-167. 